\title{
Mejoramiento del confort térmico de vivienda en uso en la ciudad de Huaraz con el aprovechamiento de la energía solar pasiva
}

Improvement of thermal confort of housing in use in the city of Huaraz with the use of passive solar energy

\section{Marco Silva Lindo $^{1}$, Richard Depaz Blácido ${ }^{1}$ y Oscar Alva Villacorta ${ }^{1}$}

\section{RESUMEN}

En la región Áncash, dadas las bajas temperaturas, es importante tener una vivienda con un adecuado comportamiento térmico que incremente el confort y salvaguarde la salud de sus habitantes; este proyecto establece como objetivo mejorar las condiciones de confort térmico en una vivienda en uso en la ciudad de Huaraz, con el aprovechamiento de la energía solar pasiva. La metodología fue experimental, con un diseño pre-experimental de pre-prueba/post-test de una vivienda. Determinó las características de temperatura y humedad relativa. Y se definieron las intervenciones a realizarse. La humedad relativa se redujo del $23 \%$ a $46 \%$, mientras que la temperatura de los ambientes aumentó en $1.2^{\circ} \mathrm{C}$ hasta $2.6^{\circ} \mathrm{C}$, obteniéndo habitaciones con una temperatura considerada como confortable en invierno que es de $20+/-2^{\circ} \mathrm{C}$. Los ambientes logran temperaturas mayores a $20^{\circ} \mathrm{C}$ por más de siete horas diarias. La implementación tiene un beneficio cuantificable en ahorro de energía eléctrica. Entre los beneficios no cuantificables de la implementación se tiene que los ocupantes de la vivienda tienen una mejor calidad de vida al tener ambientes más confortables.

Palabras clave: confort térmico; energía solar; energía solar pasiva.

\footnotetext{
1 Universidad Nacional «Santiago Antúnez de Mayolo». Huaraz. Perú.
} 


\section{ABSTRACT}

In Ancash region, given the low temperatures, it is important to have a home with adequate thermal behavior that increases the comfort and safeguard the health of its inhabitants; this project established objective of improving the conditions of thermal comfort in a house in use in the city of Huaraz, through the use of passive solar energy. The methodology was experimental, with a design of pre-test/post-test of a home. The characteristics of temperature and relative humidity were determined. Interventions were then defined to be held. Finally, the implementation was made. Subsequently, measured the temperature and relative humidity to evaluate thermal comfort.

The relative humidity was reduced to $23 \%$ to $46 \%$, while the temperature of the environment $1.2^{\circ} \mathrm{C}$ increased up to $2.6{ }^{\circ} \mathrm{C}$, resulting in rooms with a temperature considered to be comfortable in winter which is $20+/-2^{\circ} \mathrm{C}$. Environments have achieved temperatures higher than $20^{\circ} \mathrm{C}$ for more than seven hours a day. On the other hand, the implementation has a measurable benefit in electric energy saving. Among the non-quantifiable benefits of the implementation is that the occupants of the House have a better quality of life by having more comfortable environments.

Keywords: thermal comfort; solar energy; passive solar energy.

\section{ICHIKLLACHAW}

Anqash suyuqa alalaq markam, tsayraykurmi quñuq wayintsik kapamashwan llapan taakuqkuna alli kawayaananpaq; kay uryayqa rurakashqa Waras markachaw wayikunapa quñuynin alliyaananqanraykur, parwarku intipa achachayninwan. Kay rurayninqa ikpirimintalmi nishqam kashqa wayikunapa puntawan qipa chanintsay test nishqanwan. Kaychaw achachaynintawan uquynintam riqikashqa. Niykur imanaw ruraynintam rurakashqa. Puntata churakarmi qallaqashqa, qipamannam achachaynintawan uquynintam tupukashqa, quñuyninta chanintsaatsinapaq.

Uquyninqa 23\%piq 46\%kamam ichikyashqa, maa achachayninqa 1.2Cpita 2.6Ckamam witsashqa, kaywan wayikunapa quñuynin alalay killachaw $20+/-2^{\circ} \mathrm{C}$ chashqa. Kaywan $20^{\circ} \mathrm{C}$ witsayman chashqa qanchis witsaypiq punchawkunapa uurankunawan. Kaywanqa iliktrika atskita mananam alliqqa ushatsintsiknatsu. Wayikunachaw yachakuqkunapa kawayninqa alliyanmi quñuq wayinkuna kayaapunqanraykur.

Pushaq shimikuna: alli quñuy; inti achachay; parwarku inti achachay.

\section{INTRODUCCIÓN}

La ciudad de Huaraz está ubicada a 3,080 msnm, y como tal tiene un clima frígido, que se ha intensificado por el cambio climático. En esta ciudad, un gran número de 
viviendas no cuenta con sistema alguno que brinde confort térmico a sus ocupantes, comprometiendo los niveles de calidad de vida que debe tener una persona para poder ser capaz de desarrollarse y desenvolverse con comodidad en el hogar, alejando toda sensación de malestar que pueda tener consecuencias tanto patológicas como psicológicas. Para lograr el confort térmico, que permita la comodidad en el hogar, algunos pobladores recurren al empleo de calentadores eléctricos, a gas o combustible fósil, pero que involucran costos de implementación y mantenimiento permanente como el pago del recibo de energía eléctrica o el consumo de combustible, lo que ocasiona una creciente contaminación en el ambiente; muchos otros pobladores deben soportar severas condiciones de disconfort térmico, ante la imposibilidad de afrontar los gastos energéticos y por el desconocimiento de otras alternativas económicas.

El sistema energético actual está basado en una fuerte dependencia de los combustibles fósiles, que son nuestra principal fuente de energía y cuyo alto consumo está provocando un cambio climático que ya se está dejando sentir. Aunque siempre haya existido preocupaciones sobre el suministro y precio futuro de la energía, al respecto Meisen y Krumpel (2009) sostienen que los países consumidores tuvieron que modificar costumbres y buscar opciones para reducir su dependencia de fuentes no renovables.

La International Copper Association (2010) sostiene que en la década de los ochenta, aparecen evidencias de un aumento en las concentraciones de gases que provocan el efecto de invernadero en la atmósfera terrestre, las cuales han sido atribuidas, en gran medida, a la quema de combustibles fósiles.

La región Áncash dispone de un enorme potencial de recursos renovables que mayoritariamente no están siendo aún aprovechados. Es necesario contribuir a la puesta en valor de estos recursos y concienciar a los distintos agentes para que aúnen esfuerzos para conseguir un sistema energético más justo, solidario y sostenible.

El Grupo de Apoyo al Sector Rural de la Pontificia Universidad Católica del Perú (2012) ha propuesto la Casa Caliente Limpia: k'oñichuyawas; que consiste en una vivienda que cuenta con un conjunto de tecnologías apropiadas: pared caliente, cocina mejorada, terma solar y sistema de aislamiento de techos que permiten, principalmente, contrarrestar el problema del friaje en las zonas alto andinas. También ha construido La Casa Ecológica Andina en el distrito de Langui, que pretende constituir un modelo de vivienda saludable y amigable con el medio ambiente.

El Instituto General de Investigación de la Universidad Nacional de Ingeniería desarrolló el proyecto Propuesta Técnica de Confort Térmico para Viviendas en Comunidades localizadas entre 3000 y 5000 msnm, en las zonas de San Francisco de Raymina en Ayacucho y Vilcallamas Arriba en Puno. El conductor del proyecto, Espinoza (2009) concluyó que es posible elevar la temperatura del aire interior de las viviendas 
rurales alto andinas usando para ello elementos constructivos adecuados y la energía solar como fuente de calentamiento.

Alkassir y otros (2004) plantean que la percepción del ambiente térmico no depende únicamente de los parámetros ambientales, sino que es un fenómeno que incluye muchos más factores del entorno interior y exterior, del sujeto que percibe estos parámetros.

Se entiende por confort térmico, según la Cdt Chile (2008), la condición en la que las personas se sienten cómodas, es decir, en equilibrio con el ambiente térmico que las rodea. Esta condición depende de la temperatura del aire, de la temperatura de los muros del recinto habitado, de la velocidad del aire y de su humedad. Asimismo influye la vestimenta, la actividad física que se desarrolle, la alimentación, la edad, y hasta factores subjetivos como el color y tipo de decoración, entre otros. En el caso del reacondicionamiento térmico, la Cdt Chile (2010) considera que éste busca la comodidad térmica.

Encontramos que Rodríguez (2002) plantea que se puede definir como una temperatura confortable a $20+/-2^{\circ} \mathrm{C}$, valor considerado como aceptable en países desarrollados, con una humedad relativa del aire de entre $35 \%$ y $75 \%$ y un movimiento del aire inferior a $1 \mathrm{~m} / \mathrm{s}$.

Las energías renovables consisten en el aprovechamiento de los recursos naturales como el sol, el viento, los residuos agrícolas u orgánicos; son además, fuentes de energía amigables con el medio ambiente. Sobre la energía solar, Horn (2006) sostiene que es el recurso energético con mayor disponibilidad en casi todo el territorio peruano. En la gran mayoría de localidades del Perú, la disponibilidad de la energía solar es bastante grande y bastante uniforme durante todo el año.

Para que un edificio se beneficie con muy buena radiación solar, se debe tener en cuenta la energía solar en el diseño arquitectónico, condiciones que son discutidas en Jiménez (2008), como las fachadas dobles, orientación hacia el sur y superficies vidriadas, entre otros. El aislamiento térmico desempeña un papel importante para optimizar la proporción del aporte solar pasivo en calefacción y en la iluminación de un edificio. Una casa que posea energía solar pasiva estará contribuyendo a un importante ahorro energético. Para su concepción la arquitectura bioclimática recurre a la energía solar, energía eólica, de la biomasa, entre otras y al propio diseño arquitectónico. Se denomina captación solar pasiva al método de captación de la radiación solar que funciona sin necesitar aporte energético externo. También se denomina pasivo al sistema que ocasionalmente pueda utilizar un pequeño equipo para acelerar los intercambios térmicos aunque no sea imprescindible para su funcionamiento. La captación solar pasiva abarca dos tipos de elementos: los elementos captadores que recogen la radiación solar y los elementos acumuladores que son sistemas que tienen la propiedad de almacenar en su interior la energía calorífica de modo que pueda ser utilizada con posterioridad. 
El objetivo de la investigación es lograr condiciones de confort térmico en una vivienda en uso en la ciudad de Huaraz, implementando los acondicionamientos de sistemas no contaminantes y de bajo costo en la edificación, para el aprovechamiento de la energía solar pasiva que permita sustituir el consumo de energía eléctrica y mejorar las condiciones de vida de sus habitantes.

\section{MATERIALES Y MÉTODOS}

Se trata de una investigación aplicada con enfoque cuantitativo y de alcance explicativo. El diseño metodológico adoptado fue del tipo experimental de la clase de pre experimento. Se realizaron pre experimentos con un diseño de pre prueba/pos prueba con una sola vivienda.

El proceso se cumplió en las siguientes fases: Determinación de la vivienda en donde se intervendrá.

Elaboración de instrumentos para la recolección de datos y determinación de las características ambientales de la vivienda en donde se intervendrá. Toma de datos de temperatura y humedad relativa en los ambientes de la vivienda antes de la intervención, para establecer una línea de base.

Planteamiento de diseños de elementos para aplicación de las tecnologías de aprovechamiento de la energía solar de forma pasiva, a nivel de vivienda.

El diseño fue puesto a consideración de la familia beneficiaria para conocer sus puntos de vista y apreciaciones en general, las sugerencias de los ocupantes fueron implementadas reformulando el diseño inicial. Implementación de las tecnologías en la vivienda seleccionada y el seguimiento de las mismas para evaluar el rendimiento, la aceptación social de las mismas y la forma cómo evoluciona el confort térmico y la calidad de vida de los ocupantes de la vivienda.

La población y unidad de análisis fue una vivienda de albañilería confinada de dos niveles, de características promedio, en la ciudad de Huaraz, en el barrio de Pedregal Medio, en el distrito de Huaraz, cuyos ocupantes se mostraron favorables a la aplicación de las mejoras planteadas en el estudio. La vivienda tiene $150 \mathrm{~m}^{2}$, con muros de ladrillo, vigas y columnas de concreto armado, pisos de cerámico y techos aligerados de concreto armado y techos con planchas de eternit gris, pintada exteriormente con crema claro, se ubica entre dos edificaciones, construidas hacia sus lados norte y sur, y tiene su ingreso principal mirando hacia el oeste. La vivienda tiene $7.80 \mathrm{~m}$ de frontis y $17.00 \mathrm{~m}$ de largo y $6.50 \mathrm{~m}$ de altura, de forma rectangular. 


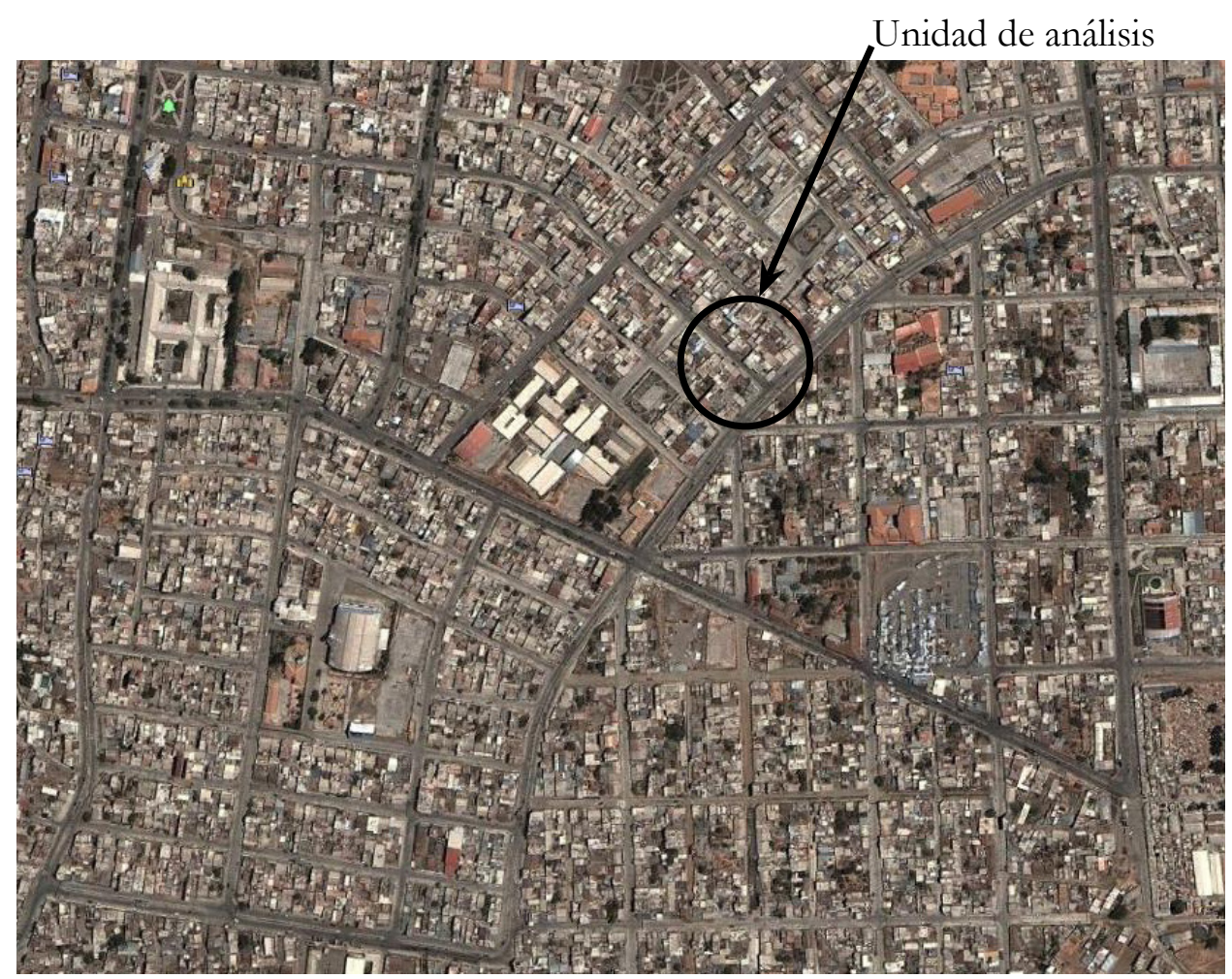

Figura 1. Ubicación de vivienda intervenida

Todo el perímetro de la vivienda está expuesto, por lo que el área implicada en las pérdidas es grande, aunque tiene el aspecto positivo de que el lado mayor recibe radiación solar durante todo el día. La vivienda se orienta principalmente de este a oeste y recibe la radiación solar por su lindero norte. La vivienda ha sido diseñada y construida ignorando la orientación geográfica terrestre, siguiendo los condicionantes urbanísticos municipales que atienden a otros criterios. El terreno es inclinado y descendente de este a oeste. La vivienda tiene vistas hacia el norte y el oeste, y en ambas tiene ventanas tanto en la primera como en la segunda planta, hacia el norte se ubica el patio de la casa.

Huaraz presenta un clima templado de montaña tropical, soleada y seca durante el día y frío durante la noche, con temperaturas medias anuales entre $13-17^{\circ} \mathrm{C}$ y máximas absolutas que sobrepasan los $22^{\circ} \mathrm{C}$ y mínimas de $3^{\circ} \mathrm{C}$.

La técnica empleada fue la observación, con el registro a través de fichas técnicas y fotografías de las características de la vivienda escogida. Las fichas técnicas se estructuraron para recopilar información sobre el comportamiento térmico de los diversos ambientes de la vivienda, durante treinta días antes y después de la intervención, para obtener datos sobre temperatura y humedad de los ambientes y del exterior. La implementación de las tecnologías de aprovechamiento de la energía solar pasiva se realizó en un marco de respeto a los usos y costumbres de los ocupantes de la vivienda intervenida. 
En el primer nivel de la vivienda, el ambiente con menor confort térmico es la sala comedor y en el segundo nivel es el dormitorio principal, que se ubican al sur de la vivienda; se aprecia que solamente reciben radiación solar en las tardes y están expuestas a flujos de aire que ingresan por la puerta y ventanas, y el balcón en el sector oeste y por el portón en el lado este, además de las puertas y ventanas del patio central de la vivienda.

Se planteó reducir la pérdida de calor evitando la circulación de aire del exterior hacia el interior aplicando el aislamiento térmico con la instalación de burletes en las rendijas de puertas y ventanas del primer piso. Lograr una captación directa e indirecta con elementos tipo invernadero que captan directamente energía en horas diurnas y pueden también acumularla en muros masivos y suelos situados frente a los elementos acristalados; para eso se convirtió el patio central, al lado norte de la vivienda, en un invernadero de $4.00 \mathrm{~m}$ x $3.90 \mathrm{~m}$ x $2.80 \mathrm{~m}$. La pared que da hacia el norte se consideró de un vidrio doble con estructura metálica de soporte y la cubierta con planchas de policarbonato sobre viguetas metálicas. Lograr una captación directa con vanos acristalados verticales con vidrios dobles con cámara de aire, lunas de baja emisividad y alto factor solar. Para el efecto se convirtió en un invernadero adosado al balcón frontal de la vivienda, en el segundo piso, que también serviría como un aislante térmico y acústico, se usó un vidrio aislante como cerramiento del balcón, de dimensiones $3.92 \mathrm{~m}$ de ancho por $2.20 \mathrm{~m}$ de alto, con estructura de soporte metálica.

\section{RESULTADOS}

Comportamiento térmico

Se ha registrado las características de humedad relativa y temperatura de los dos ambientes seleccionados de la vivienda: la sala comedor en el primer piso y el dormitorio principal en el segundo piso, antes y después de la intervención.

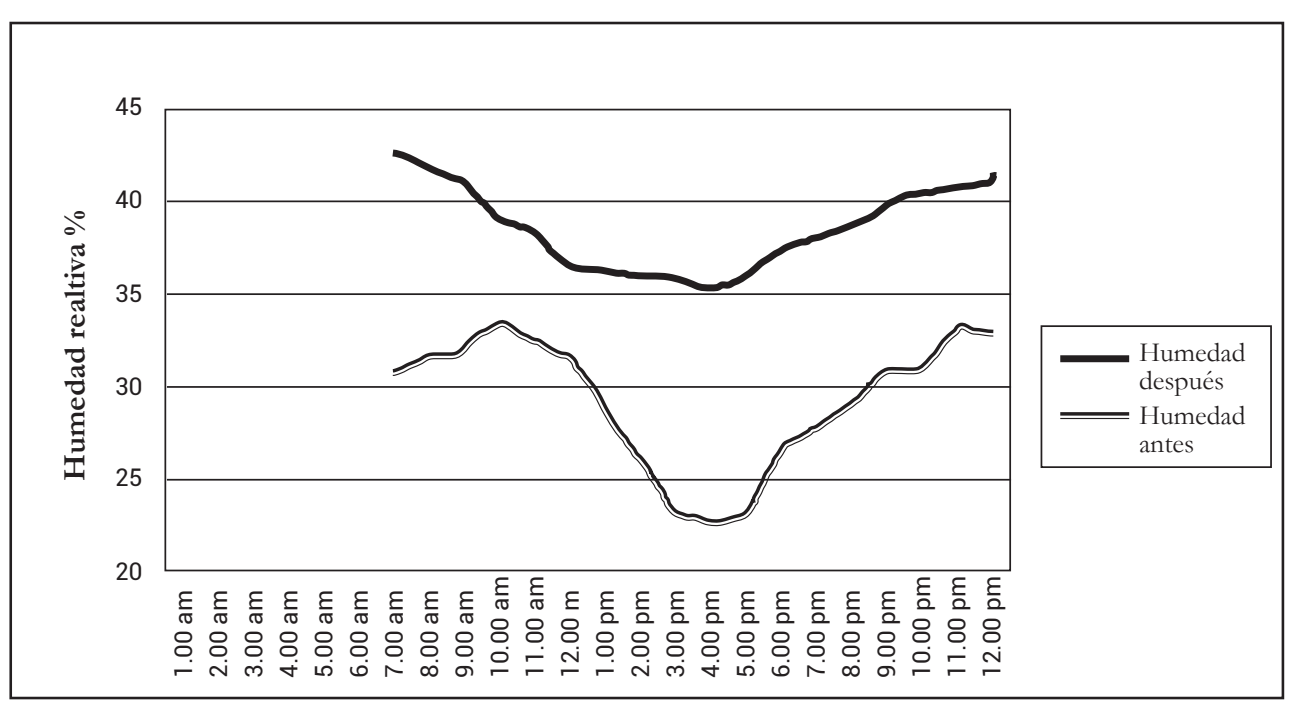

Figura 2. Humedad interior en la sala-comedor antes y después de la intervención 


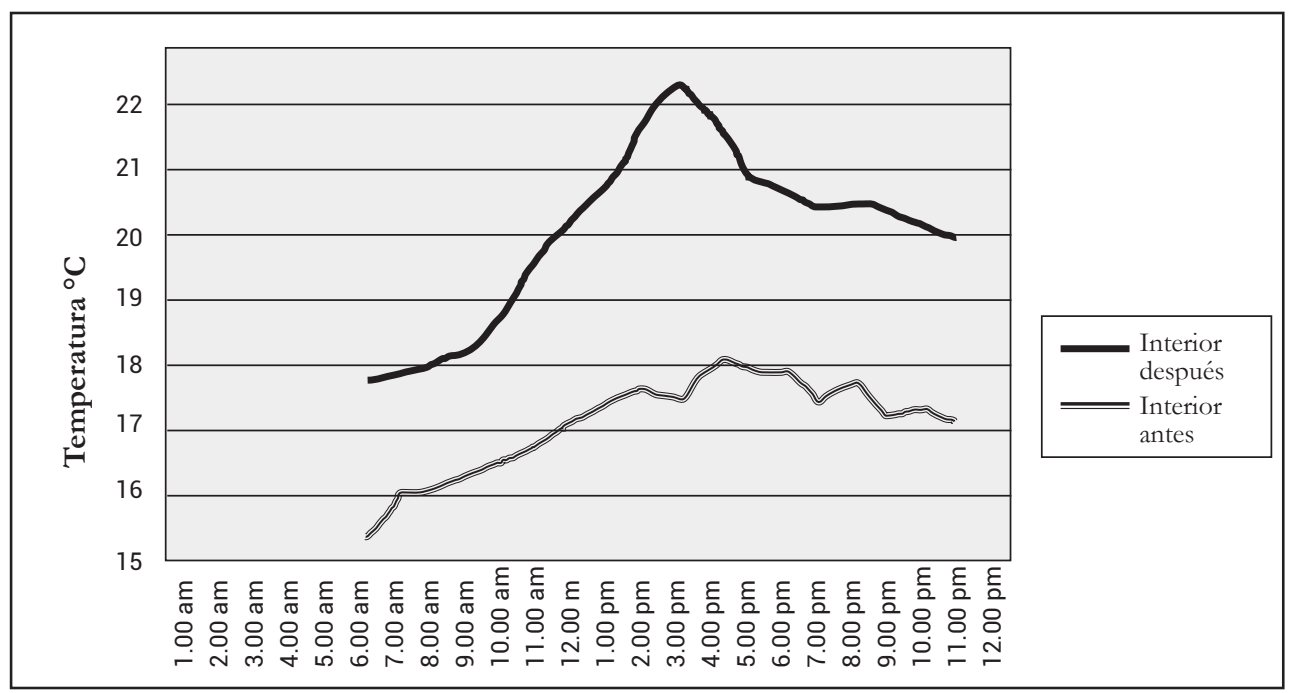

Figura 3. Temperatura interior en la sala-comedor antes y después de la intervención

En la sala comedor, la humedad relativa antes de la intervención llegaba a 43\%, mientras que después muestra valores hasta $33 \%$. La temperatura interior antes llegaba a $17.9^{\circ} \mathrm{C}$, mientras que después de la intervención llega a $21.6^{\circ} \mathrm{C}$.

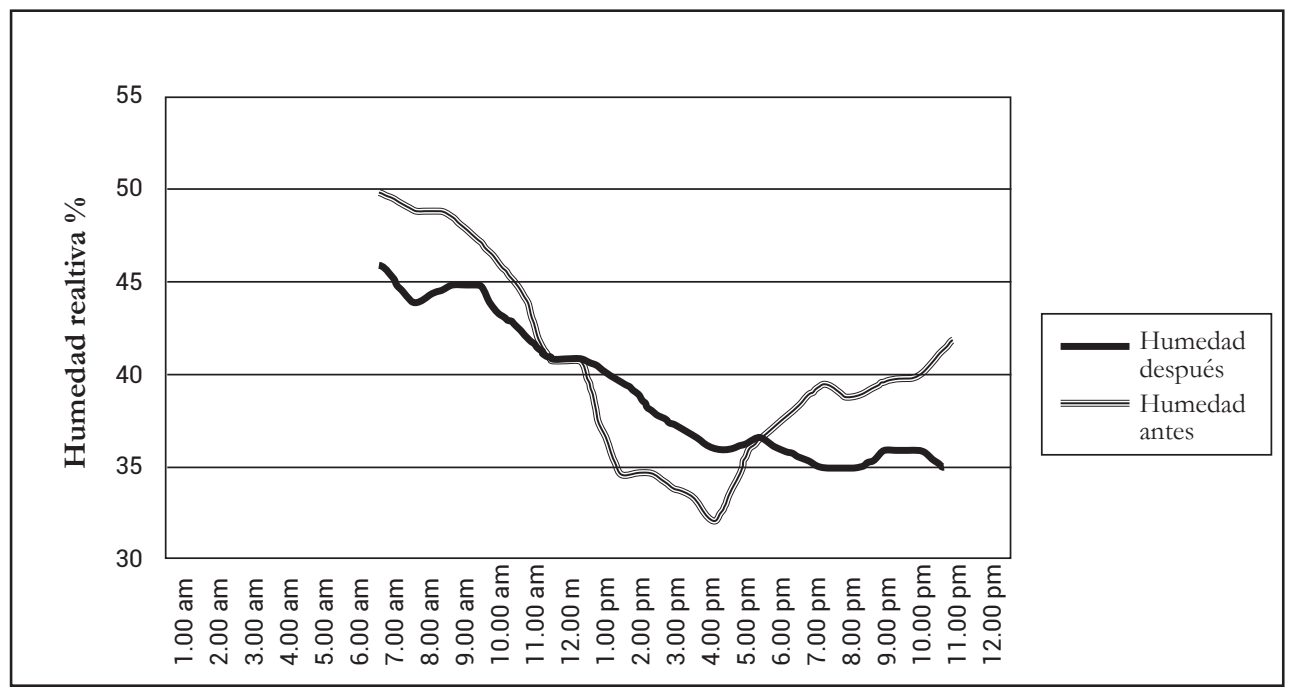

Figura 4 Humedad interior en el dormitorio principal antes y después de la intervención

Se aprecia que en el dormitorio principal, la humedad relativa antes de la intervención era de $50 \%$, mientras que después llegaba a $46 \%$. 


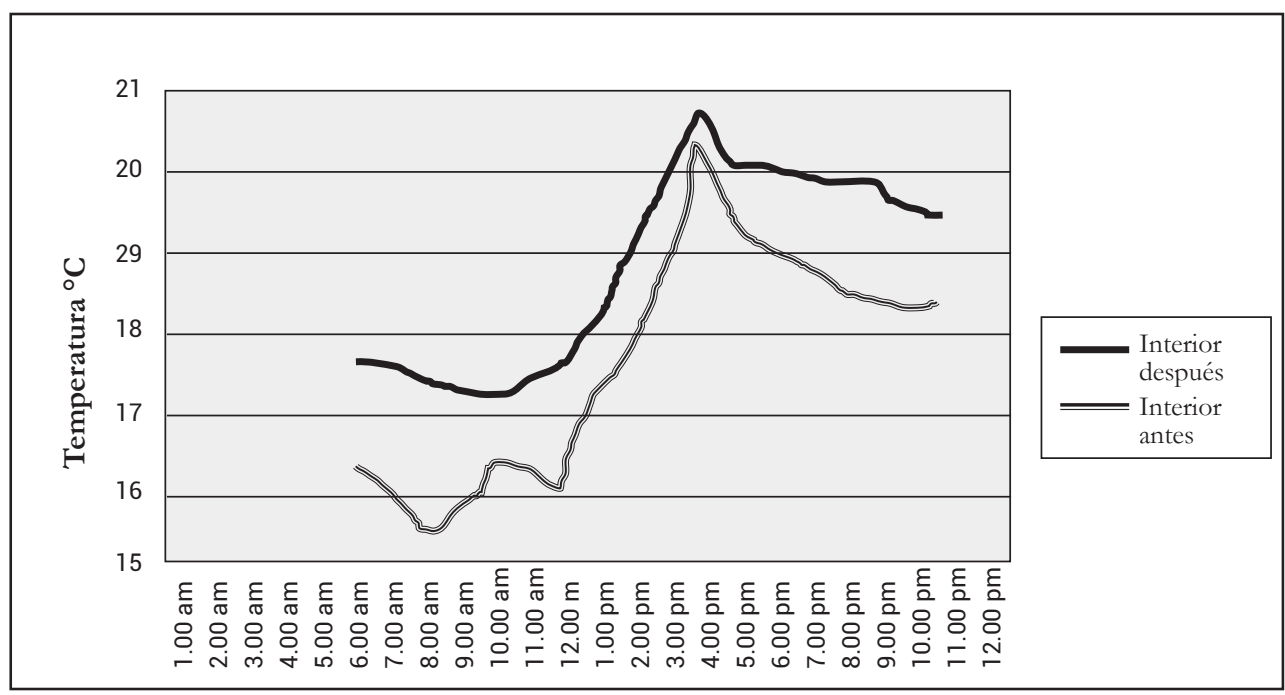

Figura 5. Temperatura interior en el dormitorio principal antes y después de la intervención

En el dormitorio principal, la temperatura interior antes de la intervención era de $20.3^{\circ} \mathrm{C}$ y después llega a $20.7^{\circ} \mathrm{C}$.

La inversión total de la intervención es de S/4,085.00 nuevos soles, que incluye los materiales y mano de obra.

El consumo eléctrico de un calentador en la sala de la vivienda es de $6.30 \mathrm{kwh}$ para el calentador durante cinco horas, lo que equivale a un gasto de $\mathrm{S} / 2.75$ nuevos soles por cada día y por punto de calefacción. Con este gasto se logra elevar en $1^{\circ} \mathrm{C}$ la habitación.

\section{DISCUSIÓN}

Los sistemas planteados e implementados, involucran a los ambientes considerados como de menor confort térmico de la vivienda intervenida y en conjunto han logrado una mejora de la temperatura y humedad interior en la edificación. Los burletes usados evitan el paso del aire del exterior al interior por las rendijas de puertas y ventanas. La conversión del patio central, en un invernadero ha logrado ganar un espacio habitable, útil para actividades recreativas, mediante el cual se puede almacenar calor y calentar por conducción los ambientes contiguos de la sala y comedor. El acondicionamiento del balcón, en el segundo piso, con vanos acristalados verticales no solo es útil para transmitir calor sino también opera como un aislante acústico. Se confirma lo señalado por Jiménez (2008), en el sentido de que se puede realizar viviendas que optimicen los recursos naturales, abasteciéndose de su propia energía.

En la Sala-Comedor la humedad relativa se ha reducido, antes de la intervención oscilaba entre $35 \%$ a $43 \%$ y ahora se tienen valores de $23 \%$ a $33 \%$, en promedio ha disminuido un $9 \%$. El invernadero adosado ahora permite que las puertas del comedor 
y sala que dan hacia él puedan estar abiertas, lo que permite mayor ventilación y una baja de la humedad. La temperatura interior ha aumentado, antes oscilaba entre $15.5^{\circ} \mathrm{C}$ a $17.9^{\circ} \mathrm{C}$, mientras que después de la intervención se obtuvo valores entre $17.6^{\circ} \mathrm{C}$ y $21.6^{\circ} \mathrm{C}$; en promedio la temperatura interior ha aumentado en $2.6^{\circ} \mathrm{C}$ y en relación a la temperatura mínima esta se ha incrementado en $2.1^{\circ} \mathrm{C}$. Antes la temperatura no llegaba a $18^{\circ} \mathrm{C}$, y ahora se ha logrado que pase los $20^{\circ} \mathrm{C}$.

Se aprecia que en el dormitorio principal, la humedad relativa presenta un menor rango de variación que antes de la intervención, antes oscilaba entre 32\% a 50\%, mientras que después oscila entre valores de $35 \%$ a $46 \%$; en promedio la humedad ha disminuido un $2 \%$ y podría decirse que se encuentra más estable que antes. La temperatura interior presenta mayores valores que los existentes antes de la intervención: antes oscilaba entre $15.5^{\circ} \mathrm{C}$ y $20.3^{\circ} \mathrm{C}$, mientras que después se obtuvo valores entre $17.2^{\circ} \mathrm{C}$ y $20.7^{\circ} \mathrm{C}$. En promedio la temperatura interior en el dormitorio principal ha aumentado en $1.2^{\circ} \mathrm{C}$, y en relación a la temperatura mínima esta se ha incrementado en $1.7^{\circ} \mathrm{C}$. Antes la temperatura llegaba por debajo de $17^{\circ} \mathrm{C}$, y ahora en ningún momento del día se llega a esa temperatura.

De acuerdo a lo señalado por Rodríguez (2002), los ambientes de la vivienda intervenida pueden considerarse confortables térmicamente, pues la temperatura interior se encuentra en $20+/-2^{\circ} \mathrm{C}$, y la humedad en el rango de $35 \%$ a $75 \%$ en el caso del dormitorio principal; pero no así en la sala-comedor donde está entre $23 \%$ a 33\%, aunque en general los ocupantes de la vivienda perciben una mejora en las condiciones térmicas de su hogar.

Espinoza (2009) determinó para una vivienda de adobe en la localidad de San Francisco de Raymina, ubicada en la región Ayacucho a $3700 \mathrm{msnm}$, que la temperatura promedio del aire aumentó de $3^{\circ} \mathrm{C}$, antes de la modificación hasta $9^{\circ} \mathrm{C}$ después de la modificación; esto implica una elevación de las temperaturas mínimas de $6^{\circ} \mathrm{C}$ que es mayor a las obtenidas en el presente estudio para una vivienda de ladrillo. La diferencia puede deberse al material de las viviendas.

Algunos beneficios pueden ser cuantificables (ahorro de combustibles, menor contaminación del medio ambiente, etc.) y otros difícilmente ponderables (mejor calidad de vida, vivir en ambientes más confortables en invierno y en verano, situaciones que tienen que ver indirectamente con la mayor productividad).

La inversión total de la intervención fue de S/4,085.00 nuevos soles, que incluye los materiales y mano de obra. En caso de que se deseara mejorar en $1^{\circ} \mathrm{C}$ la temperatura en tres ambientes: sala, comedor y un dormitorio, tendría que gastarse $\mathrm{S} / 2.75$ por día y por ambiente, lo que implica un gasto diario de $\mathrm{S} / 8.25$, un gasto mensual de $\mathrm{S} / 250.94$ y un costo anual de $\mathrm{S} / 3,011.25$. Por lo tanto la inversión se recupera en 17 meses, de ahí en adelante no existirían gastos hasta por un período mínimo de 10 años. 


\section{CONCLUSIONES}

La humedad relativa de los ambientes se reduce y estabiliza, evitando los mínimos y máximos de antaño, obteniendo que oscilen de $23 \%$ a $46 \%$, porcentaje cercano a los rangos recomendables del 35\% al 75\%.

Con la intervención se ha logrado un aumento en la temperatura de los ambientes de la vivienda intervenida, con aumentos de $1.2^{\circ} \mathrm{C}$ y hasta de $2.6^{\circ} \mathrm{C}$ en promedio, obteniendo que las habitaciones tengan una temperatura de $20+/-2^{\circ} \mathrm{C}$ considerada como confortable en invierno. Los ambientes han logrado temperaturas mayores a $20^{\circ} \mathrm{C}$ por más de siete horas.

La implementación del aprovechamiento de la energía solar pasiva en la vivienda tiene un beneficio cuantificable en ahorro de energía eléctrica, tangible después del período de 17 meses de recuperación de la inversión, sin gastos de ahí en adelante hasta por un período mínimo de 10 años.

Entre los beneficios no cuantificables de la implementación se tiene que los ocupantes de la vivienda alcanzan una mejor calidad de vida al tener ambientes más confortables, lo que se traduce en personas más contentas con su vivienda.

\section{REFERENCIAS BIBLIOGRÁFICAS}

Alkassir, Awf y otros. 2004. Estudio Experimental de las condiciones del Confort Térmico. Badajoz: Universidad de Extremadura - Escuela de Ingenierías Industriales.

Corporación de Desarrollo Tecnológico de la Cámara Chilena de la Construcción. 2008. Manual Técnico Aislación térmica exterior. Santiago de Chile: Corporación de Desarrollo Tecnológico de la Cámara Chilena de la Construcción Chile.

Corporación de Desarrollo Tecnológico de la Cámara Chilena de la Construcción. 2010. Reacondicionamiento térmico de viviendas en uso. Santiago de Chile: Corporación de Desarrollo Tecnológico de la Cámara Chilena de la Construcción Chile.

Espinoza, Rafael. 2009. Propuesta Técnica de Confort Térmico para Viviendas en Comunidades localizadas entre 3000 y 5000 msnm. Lima: Centro de Energías Renovables, Universidad Nacional de Ingeniería.

Horn, Manfred. 2006. El estado actual del uso de la energía solar en el Perú. Los retos energéticos del Perú. Lima:, Volumen XXIX, Nr.11.

International Copper Association. 2010. Energías Renovables para la Generación de Electricidad en América Latina: Mercado, tecnologías y perspectiva. Santiago de Chile: Copper Connects Life.

Jiménez, Edgar. 2008. Estrategias de Diseño para brindar confort térmico en vivienda en la ciudad de Loja. Loja: Tesis de Arquitectura. Universidad Técnica Particular de Loja. 
Meisen, Piter y Krumpel, Sebastián. 2009. El potencial de América Latina con Referencia a a Energía Renovable. San Diego: GENI Global Energy Network Institute.

PUCP. 2012. Koñichuyawasi: Casa caliente limpia. Lima: Pontificia Universidad Católica del Perú. <http://gruporural.pucp.edu.pe/proyectos/ konichuyawasi -casa-caliente-limpia $>$ [Consulta: 07-09-2012]

Rodríguez, Gabriel. 2002. «Temperatura de Confort». Revista BIT N² 27. Santiago de Chile: Corporación de Desarrollo tecnológico Cámara Chilena de la Construcción. 25-26.

Fecha de recepción: 08 de enero 2016

Fecha de aceptación: 24 de mayo 2016

\section{Correspondencia}

Marco Silva Lindo

maslindo1@yahoo.com 